\title{
Performance Limit of Infra-Red Thermomechanical Imager with Optical Readout
}

\author{
V.A. Fedirko ${ }^{1,2, *}$, E.A. Fetisov ${ }^{1}$, K.K. Svidzinsky ${ }^{1}$ \\ ${ }^{1}$ National Research University of Electronic Technology "MIET" (MIET), Moscow, Russia \\ ${ }^{2}$ Moscow State University of Technology "Stankin” (MGTU “Stankin”), Moscow, Russia \\ *Corresponding Author: vfed@mail.com
}

Copyright $@ 2014$ Horizon Research Publishing All rights reserved.

\begin{abstract}
We analyze the performance limit of uncooled thermo-mechanical infra-red imagers with optical readout. We have shown that for large enough frame format thermal noise of load resistance of output electronic cascade of optical readout system determines mainly the noise equivalent temperature difference, NETD, of such systems. For middle frame format they can successfully compete with micro-bolometer infra-red detectors. Higher power low noise light source for optical readout system and more exquisite phase detection enable them to be competitive in large frame format too.
\end{abstract}

Keywords Uncooled Infra-Red (IR) imager, Bimaterial Thermomechanical Sensor, Optical Readout, NETD, Performance Limit, MEMS Technology

\section{Introduction}

Micromechanical uncooled infra-red (IR) image sensors for long wave infrared radiation $(8-14 \mu \mathrm{m})$ with optical readout have been intensively studied lately [1-6]. They are developed as an alternative to industrially produced, but yet rather expensive micro-bolometer IR detectors [7]. The latter require complex combined MEMS/CMOS technology to form readout integrated circuit under micro-bolometer matrix which is to be thermally-isolated from substrate.

The distinctive feature of an optical readout IR imager (ORI) is the use of an optical readout scheme for IR imaging instead of commonly used electrical readout. The IR sensor array is electrically passive so it has no self-heating, and the system has low energy consumption. It needs no cooling and may operate at rather high temperature and in enhanced radiation background, and it has high linearity, including also detection in bands beyond LWIR $(8-14 \mu \mathrm{m})$, e.g. MWIR $(3-15 \mu \mathrm{m})$, dualband and $\mathrm{THz}(>14 \mu \mathrm{m})$. Standard MEMS technology on large format substrate wafer processing can be applicable for FPA fabrication so the IR ORI systems are expected to be low-cost, perhaps, twice lower, than bolometric IR detectors.
The principal scheme of IR ORI is shown in fig.1. The basic element of IR ORI is a thermo-mechanical focal plane array (TMFPA) of micro-mirrors suspended on thermally sensitive bimaterial micro-cantilevers. The cantilevers shift (or turn) the mirrors bending due to the thermal heating. The IR object radiation is focused by IR optics onto TMFPA and forms the temperature image of the object on the FPA input surface which is replicated into the micro-mirrors relief on the FPA output surface created by their displacement due to bimorph micro-cantilevers bending under distributed heating. An optical readout system (ORS) with cold light source, say laser diode (LD), replicate the FPA mirrors relief to the phase relief of the optical wave reflected from modulated mirrors panel. The phase-modulated pattern is then transformed to the optical amplitude-modulated pattern using optical filtration, and is projected to charge coupled device (CCD) panel or some other detector through imaging optics.

Noise equivalent temperature difference (NETD) is a key figure of merit for an IR imager which determines its performance limit. NETD values of $500 \mathrm{mK}$ have been reported for medium format TMFPA (256 x 256) ORI [1], and $100 \div 200 \mathrm{mK}$ for small format imagers $(\sim 100 \times 100)$ $[3,4]$. That is about three times worse than NETD values for the micro-bolometer FPA sensors of the same size. It is worth noting that the above experimental values are more than an order worse than their theoretical estimations, but we failed to find any explanation for that discrepancy in literature. Nevertheless thermo-mechanical infra-red imagers with optical readout are considered as potentially advantageous as compared to micro-bolometer detectors because of format scale extension (up to 2000x2000 after $[2,5])$ without loss of sensitivity and for economic reason. However to realize these potential advantages the ORI performance limit must be brought to the level compared to that of bolometer sensors.

The aim of our work is to find an approach to solve that problem. The above mentioned unexplained gap between calculated and measured NETD values available for cited ORI system gives some hope for that. 


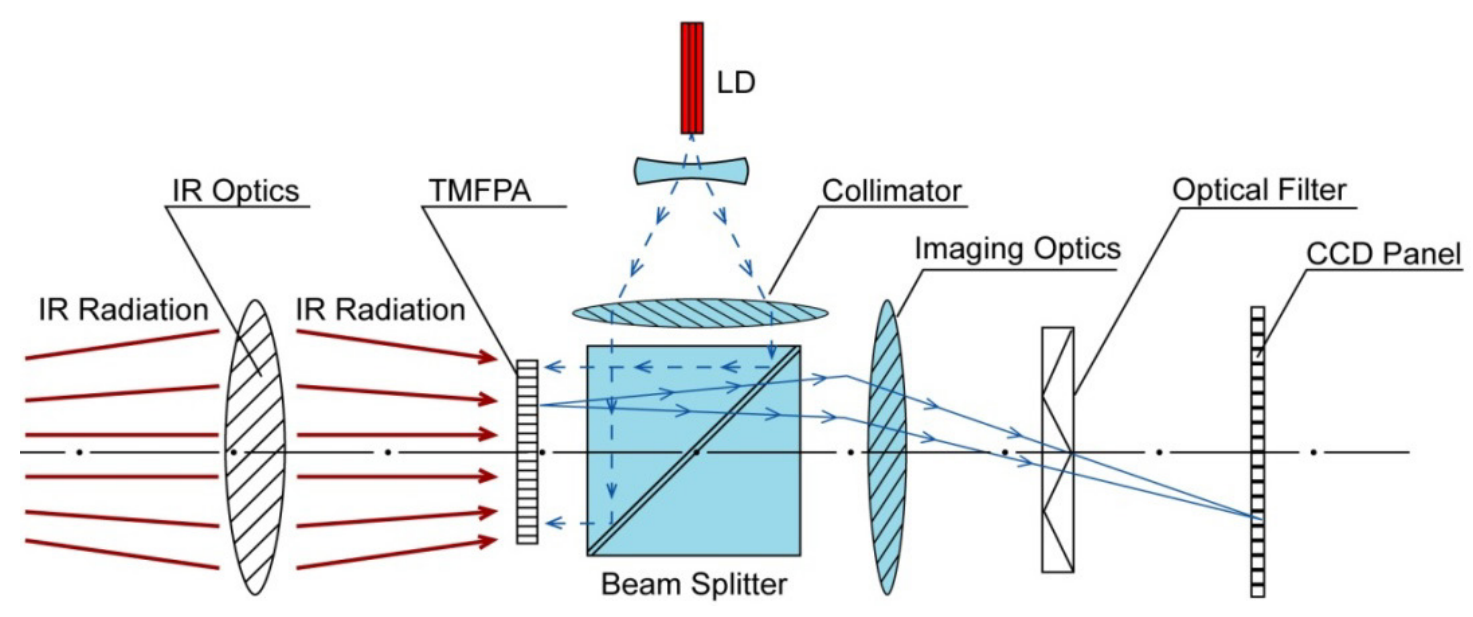

Figure 1. Principal Scheme of IR ORI

We shall focus on studying the ORI's NETD dependence on the number of OMFPA elements $\mathrm{N}$, i.e. on image resolution which is the fundamental characteristic of the system. Based on the results of that study we shall discuss some thinkable ways of optimizing optical readout system to reduce ORI's NETD.

\section{Noise of Optical Readout System}

As seen from Fig.1, the IR radiation from an object segment $\Delta A$ is projected on some thermo-mechanical (TM) pixel of $m \times n$ FPA matrix, and heat its detector by the temperature $\Delta T_{\mathrm{D}}$ proportional to the segment excess temperature, $\Delta T_{\mathrm{O}}$, over background temperature:

$$
\Delta T_{\mathrm{D}}=S_{T} \cdot \Delta T_{\mathrm{O}}
$$

where $S_{T}$ - can be considered as the temperature sensitivity of a TM element of FPA.

The $\Delta T_{D}(i j)$, as an inner input signal for (ij) TM pixel, is transformed and transferred by ORS to the CCD photoelectric detector which produces the output photocurrent:

$$
I(i j)=K \cdot \Delta T_{D}(i j),
$$

$K$ being the ORS internal transfer factor. Here we have taken into account, that format of the CCD panel must be no less than $N$ to keep the TM FPA resolution, so CCD panel format $m \times n=N$ is an optimal one for ORS.

Output current noise, consists of the TM element noise which is read by ORS and the ORS own noise $[8,9]$. TM element noise was well analyzed elsewhere (see e.g. [4,5, $8-10]$ ) and its estimations seem relevant. In contrast, the ORS noise needs, in our view, the closer analysis

Main ORS noise sources which contribute to the output photocurrent noise $\delta I(i j)$ are as follows:

- photo-induced current shot noise, $I_{S}$;

- relative intensity noise (RIN) of the light source (LD), $I_{R I N}$

- thermal (Johnson) noise caused by the amplifier in the
CCD output section, $I_{R}$.

We do not take into account thermal charge fluctuations and $1 / f$ noise in CCD elements as they are effectively reduced by double correlating sampling [11]. We also omit mechanical vibration-deformation noise - it results in fluctuations of phase-modulated pattern, but their impact can be minimized by proper choice of phase pattern detection technique, robust to optical path variations [1-6, $8]$.

The first three sources being statistically independent contribute additively to the total mean-square fluctuation:

$$
\left\langle\delta I^{2}\right\rangle=I_{S}^{2}+I_{R I N}^{2}+I_{R}^{2}
$$

Here

$$
I_{S}^{2}=2 e I \Delta f ; I_{R I N}^{2}=R I N \cdot I^{2} \Delta f ; I_{R}^{2}=\frac{4 k_{B} T}{R} \cdot B
$$

are well-known expressions for mean-square shot noise, RIN and Johnson noise respectively, where $I$ is the photocurrent read from a single CCD FPA element, $\Delta f$ is CCD half-frame mode frequency band, $B$ is the measurement bandwidth, $R$ is the load resistance of the CCD output electronic cascade, and $k_{B}$ is the Boltsman constant.

After [9], the ORS noise is assumed to be dominated by the shot noise of the photodetector, while other noise sources can be ignored. On that assumption the ORS noise has been estimated as negligible in total system noise compared to the FPA pixel noise. That assumption is however not fully justified. It is well known that shot noise depends strongly on exposition (see e. g., [12]), as well as RIN noise, while electrical Johnson noise does not. As an exposition per a CCD panel element diminishes with $N$, shot noise and RIN noise also diminish, so they dominate for small format FPAs only, while for large enough $N$ the assumption of [9] gets invalid. Thereby we will reexamine the ORS noise estimations in more details.

To evaluate and compare the terms in (3) note, that the output photocurrent per pixel $I \equiv I(i j)$ is proportional to the 
total light source power $P_{L}$ and inversely proportional to the total number of elements (pixels) $N$, so:

$$
I(i j)=\beta \frac{P_{L}}{N} ; \quad \beta=r \eta \cdot(e \lambda / 2 \pi \hbar c)
$$

where the proportionality factor $\beta<1$ can be treated as an average efficiency of ORS, $\lambda$ being the light wavelength, $r$ the micro-mirror reflectivity, $\eta-$ the photodetector quantum efficiency.

To estimate $I_{R I N}$ in (4) we use RIN $\approx-(120 \div 130) d B / H z$ of low noise laser diodes, developed for DVD [13]. As the frame frequency is no less than $25 \mathrm{~Hz}, \Delta f \geq 50 \mathrm{~Hz}$.

It is expedient to choose the measuring bandwidth dependent on image format to reduce the thermal noise, that is $B=N \cdot \Delta f$. Then the load resistance is to be chosen from the requirement:

$$
R=\frac{1}{2 \pi C N \Delta f}
$$

and for $I_{R}$ we find:

$$
I_{R}=2 \Delta f \sqrt{2 \pi C k_{B} T} \cdot N
$$

where is $C$ is the capacitance of the cascade. In typical CCDs, this capacitance would be in the $10 \mathrm{fF}$ to $50 \mathrm{fF}$ range [14]. (So, e.g., for full frame format $N \sim 3 \cdot 10^{5}$ with $C=10 f F$ eq. 8 gives $R \approx 1 M \Omega$.)

Further on we are interested in noise dependence on frame format $N$ and light source power $P_{L}$. From (4-7) we find for $r m s$ values of noise currents:

$$
\begin{aligned}
& I_{S} \approx 8 \cdot 10^{-11} \cdot \sqrt{\frac{P_{L}}{1 m W}} \cdot N^{-1 / 2} ; \\
& I_{R I N} \approx 8.94 \cdot 10^{-10} \cdot\left(\frac{P_{L}}{1 m W}\right) \cdot N^{-1} ;\left(R I N=10^{-13}\right) ; \\
& I_{R} \approx 1,61 \cdot 10^{-15}\left(\frac{C}{10 f F}\right)^{1 / 2} \cdot N ;
\end{aligned}
$$

where currents are measured in $A$, and we put $\beta \approx 0.4$ which is a reasonable estimation for its maximum achievable value with $r=0.7$ for $\lambda=0.78 \mu \mathrm{m}$ and $\eta=0.9$ [15].

The dependence on $N$ of all three terms in (3) for $P_{L}=1$ $m W$ - typical LD power used now in ORS, is presented in Fig. 2 in logarithmic scales.

One can see that the three lines intersect at $N \approx\left(10^{2} \div 10^{3}\right)$ where all three noise sources contribute about equally in NETD. For $N>1000$ thermal noise $I_{R}$ dominates, and it exceeds others. Only in small frame format ORI shot noise and RIN dominate where they have close values. It worth noting that shot noise predominates in neither small nor large format ORI contrary to widespread belief, though never justified.

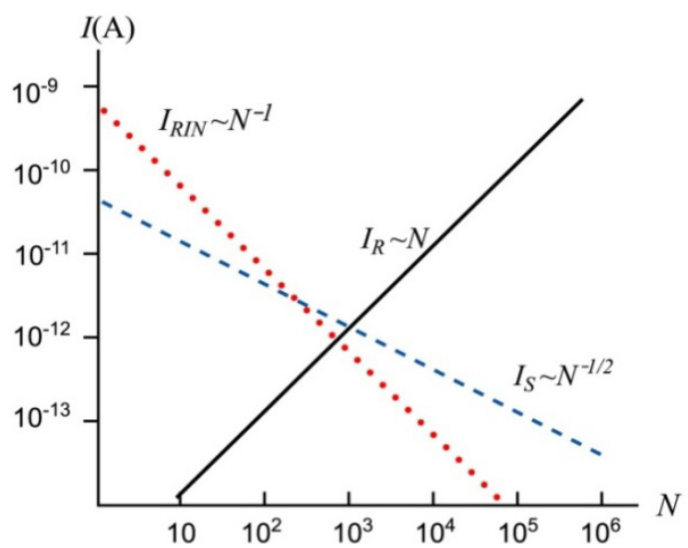

Figure 2. Noise current dependence on number of TMFPA elements: $I_{S}-$ shot noise; $I_{\mathrm{RIN}}-$ LD RIN induced noise; $I_{\mathrm{R}}-$ Johnson noise

\section{NETD Calculation}

We start from detector noise equivalent temperature difference related to the TM pixel detector of an ORI, $\mathrm{NETD}_{\mathrm{D}}$. Then for the NETD related to the object we have according to eq. (1):

$$
N E T D=S_{T}^{-1} \cdot N E T D_{\mathrm{D}}
$$

The total $\mathrm{NETD}_{\mathrm{D}}$ of an ORI is the rms sum of $\mathrm{NETD}_{\mathrm{PD}}$ due to the thermo-mechanical pixel element inherent fluctuations (i.e. thermo-mechanical fluctuations, temperature fluctuations, etc.) $[4,5,8-10]$ and NETD $_{\text {ORSD }}$ associated with ORS noise sources, considered above in section 2:

$$
N E T D_{D}=\sqrt{\left(N E T D_{P D}\right)^{2}+\left(N E T D_{O R S D}\right)^{2}} .
$$

According to theoretical estimates $[4,5,10]$, the internal NETD $_{\mathrm{PD}}$ of thermo-mechanical element can be reduced up to a value about $0.3 \mathrm{mK}$ which was confirmed experimentally. The maximum value of $S_{T}$ for such level of $\mathrm{NETD}_{\mathrm{PD}}$ is $S_{T} \approx 0.03$. One easily finds from (9) that in the lack of other noise sources the ORI NETD figure would be about $10 \mathrm{mK}$, though real values observed are, as mentioned above, more than 10 times higher. It is reasonably to believe that the gap is bridged by the ORS noise, which, as we have seen above, is dominated by the third term in (8) for practically interesting large enough frame format.

From eq. (2) we have:

$$
\left\langle\delta I^{2}\right\rangle=K^{2} \cdot\left(N E T D_{\mathrm{D}}\right)^{2}
$$

The ORI total internal transfer factor $K$ of eq. (2) can be found in linear approximation as a product of intermediate transfer coefficients on each transformation step of $\Delta T_{\mathrm{D}}(i j)$ into $I(i j)$ (see fig. 1 and comments thereafter): 


$$
\left.K=\left(\frac{I(i j)}{\Delta T_{\mathrm{D}}(i j)}\right)=\left(\frac{I(i j)}{\Delta P_{R}(i j)}\right) \cdot\left(\frac{1}{P_{R}(i j)} \cdot \frac{\Delta P_{R}(i j)}{\Delta \phi(i j)}\right)\right) \cdot\left(\frac{P_{R}(i j)}{P_{L}}\right) \cdot\left(\frac{\Delta \phi(i j)}{\Delta Z(i j)}\right) \cdot\left(\frac{\Delta Z(i j)}{\Delta T_{\mathrm{D}}(i j)}\right) \cdot P_{L} .
$$

Here $\Delta Z / \Delta T_{\mathrm{D}}=S_{T M}$ is the thermo-mechanical sensitivity of a bimorph thermo-mechanical element, $P_{R}(i j)=r P_{L} / N$ is the fraction of LD power $P_{L}$, reflected from $(i j)$ element of FPA, $\Delta \phi / \Delta Z=4 \pi / \lambda, \Delta P_{\mathrm{R}}(i j) / \cdot \Delta \varphi \cdot P_{\mathrm{R}}(i j)=\gamma<1$ is the phase detection efficiency, $I(i j) / \Delta P_{R}(i j)=\eta \cdot(e \lambda / 2 \pi \hbar c)$. Thus one has:

$$
K=\frac{2 \operatorname{er\gamma } \eta S_{T M}}{\hbar c} \frac{P_{L}}{N}
$$

With (13) we then find from (11):

$$
N E T D_{O R S D}=K^{-1} \sqrt{\left\langle\delta I^{2}\right\rangle}=\frac{\hbar c}{2 e r \eta S_{T M}} \cdot \frac{N}{\gamma P_{L}}\left\langle\delta I^{2}\right\rangle^{1 / 2} .
$$

For practically interesting frame format $N>1000$ one may put (see fig. 2):

$$
\left\langle\delta I^{2}\right\rangle^{1 / 2} \approx I_{R}
$$

and with (6) we finally come to the estimate:

$$
N E T D_{O R S D} \approx I_{R} / K=\frac{\hbar c \sqrt{2 \pi C k_{B} T} \Delta f}{e r \eta S_{T M}} \cdot\left(\frac{N^{2}}{\gamma P_{L}}\right)
$$

and

$$
N E T D \approx \frac{N E T D_{O R S D}}{S_{T}}=\frac{\hbar c \sqrt{2 \pi C k_{B} T} \Delta f}{e r \eta S_{M}} \cdot\left(\frac{N^{2}}{\gamma P_{L}}\right)
$$

where $S_{M}=S_{T} \cdot S_{T M}$. To estimate the value of transfer factor $K$ and its dependence on $N, P_{L}$, and $\gamma$ we use the typical value $r=0,7$, maximum reported $S_{T M}=0.8 \mu m / K$ [8], above mentioned $S_{T}=3 \cdot 10^{-2}$, the lowest $C=10 p F$ (see e.g. [14]) and the best value $\eta=0.9$ for available commercial CCD [15]). Then we find:

$$
K \approx 5.07 \cdot \frac{\gamma P_{L}}{N}(A / K)=5.07 \cdot \frac{\gamma P_{L}}{N} \cdot 10^{-6}\left(\frac{P_{L}}{1 m W}\right)\left(\frac{A}{m K}\right)
$$

and

$$
\begin{gathered}
\operatorname{NETD}_{\text {ORSD }}(m K) \approx 3.18 \cdot 10^{-10} \cdot N^{2}\left(\frac{\gamma P_{L}}{1 m W}\right)^{-1} \\
N E T D(m K) \approx 10^{-8} N^{2}\left(\frac{\gamma P_{L}}{1 m W}\right)^{-1}
\end{gathered}
$$

One can easily see that for $N \approx 10^{4} \mathrm{NEDT}_{\mathrm{ORSD}}$ has about the same value as the abovementioned theoretical estimation for $\mathrm{NETD}_{\mathrm{DP}} \approx 0.3 \mathrm{mK}$, if $P_{L}=1 \mathrm{~mW}$ even for maximum possible $\gamma=1$. So for real ORSI with $\gamma<1$ ORS noise dominates for image format $N>10^{4}$ and exceeds thermo-mechanical element noise more than an order of magnitude.

\section{Discussions}

Formulae (19) and (20) can explain the existing discrepancy between NETD measured in real ORI and simplistic estimations which, as one can see now, proves too rough. Besides, those formulae indicate strong NETD dependence on $N$, which has not been considered yet $[1,2$, $6,9]$. And alongside with that they clearly show that ORI NETD can be reduced by using more powerful readout light source and by optimizing the readout system to increase phase detection efficiency $\gamma$.

The phase detection efficiency limit $\gamma=1$ in principal also can be nearly achieved. Deflection readout systems with simple filters like a "slit" or "knife edge" still have been most commonly used until now. Their efficiency however can't exceed values $\gamma \approx 0.1$ as follows from diffraction theory. On the other hand, interference type filters may provide much higher efficiency, potentially close to the maximum value. Reported system of that kind [5] has rather low $\gamma$ but it is because it is not optimal. Nevertheless we believe that optimization of interference elements allows to achieve $\gamma$ value at least about 0.8 . As a possible option Fresnel biprism can be used as an effective interference filter which provides almost complete overlap of the interfering waves.

Another chance is given by the progress in development of semiconductor lasers for DVD [16]. Nowadays commercially available low noise lasers have output power increased up to $10 \mathrm{~mW}$ [17]. So, NETD of the ORI can be expected to be about 1 times reduced as follows from eq. (20) as RIN and shot noise still can be neglected. Further progress inspires hope that quite probably the low noise lasers with higher output power will be developed in near future.

NETD estimated according to (20) for some values of $\gamma$ and $P_{L}$ are presented in Table 1 which illustrates the impact of these factors on performance limit of TM ORI.

Table 1. NETD estimations for various format of TM IR ORI

\begin{tabular}{|c|c|c|c|c|}
\hline Format, $N$ & $\gamma=0.1, P_{L}=1 \mathrm{~mW}$ & $\begin{array}{c}\gamma=0.1, P_{L}=10 \\
\mathrm{~mW}\end{array}$ & $\begin{array}{c}\gamma=0.8, \\
P_{L}=1 \mathrm{~mW}\end{array}$ & $\begin{array}{c}\gamma=0.8, P_{L}=10 \\
\mathrm{~mW}\end{array}$ \\
\hline $160 \times 120=19200$ & $\approx 37 \mathrm{mK} *)$ & $\approx 3.7 \mathrm{mK} *)$ & $\approx 4.6 \mathrm{mK} *)$ & $\approx 0.46 \mathrm{mK} *)$ \\
\hline $320 \times 240=76800$ & $\approx 590 \mathrm{mK}$ & $\approx 59 \mathrm{mK}$ & $\approx 74 \mathrm{mK}$ & $\approx 7.4 \mathrm{mK} *)$ \\
\hline $640 \times 480=307200$ & $\approx 9 \mathrm{~K}$ & $\approx 0.9 \mathrm{~K}$ & $\approx 1.1 \mathrm{~K}$ & $\approx 110 \mathrm{mK}$ \\
\hline
\end{tabular}

${ }^{*}$ In that case other noise sources are also essential, including thermo-mechanical fluctuations. 


\section{Conclusions}

We have shown that the thermal noise of an ORS photodetector dominates total noise figure of full frame TM ORI, contrary to common misconception that shot noise does. That's why the performance limit of TM ORI is strongly dependent on its format and is worse than existing theoretical estimates. According to formulae (19), (20) its noise equivalent temperature difference NETD increases as $N^{2}$ with format extension. We have shown that NETD can be reduced significantly by reasonable enhance of low noise light source power of optical readout system and by use more exquisite approach to increase phase detection efficiency of ORS.

With that, the performance limit of uncooled infra-red thermo-mechanical IR imagers with optical readout less than $20 \mathrm{mK}$ can be achieved for medium format imagers which is comparable with micro-bolometer imaging systems. But TM ORIs are potentially cheaper as their technology is much simpler, and have some technical advantages. However for higher format TM ORIs more powerful low noise coherent light sources are needed to keep low NETD.

\section{Acknowledgements}

The work is supported by the Russian Ministry of education and science of (Contract No 14.430.12.0004).

\section{REFERENCES}

[1] N. Lavrik, R. Archibald, D. Grbovic, S. Rajic, P. Datskos, Uncooled MEMS IR imagers with optical readout and image processing, Proc. SPIE, Vol. 6542, 65421E, 2007.

[2] N. V. Lavrik, D. Grbovic, S. Rajic, P. G. Datskos, D. Forrai, E. Nelson, J. Devitt and B. McIntyre. Uncooled infrared imaging using bimaterial microcantilever arrays, Proc. SPIE 6206, 62061, 2006.

[3] C.D.W. Jones, C.A. Bolle, R. Ryf at al. MEMS thermal imager with optical readout, SENSORS AND ACTUATORS, A/1, 47 - 57, 2009.

[4] M. Erdtmann, L. Zhang, G. Jin, S. Radhakrishnan, G. Simelgor, and J. Salerno. Optical readout photomechanical imager: from design to implementation, Proc. of SPIE, Vol.
7298, 72980I-1, 2009.

[5] Yufeng Jin, Xiaomei Yu, Yongjun Zheng, Yuejin Zhao, Xiaohua Liu and Liquan Dong. Development of infrared FPA using bimaterial microcantilever arrays, Proc. SPIE, Vol. 8191, 819102, 2011.

[6] Yan-mei Kong, Rui-wen Liu, Bin-bin Jiao and Da-peng Chen, "Infrared camera based on optical-readout bi-material FPA", Proc. SPIE, Vol.8193, 81930X, 2011.

[7] Niklaus, Frank; Vieider, Christian; Jakobsen, Henrik. MEMS-based uncooled infrared bolometer arrays: a review. Proceedings of the SPIE, Vol. 6836, pp. 68360D (2008).

[8] Yang Zhao, Minyao Mao, R. Horowitz, A. Majumdar, J. Varesi, P. Norton, J. Kitching. Optomechanical uncooled infrared imaging system: design, microfabrication, and performance, Journal of Microelectromechanical Systems, Volume 11,Issue 2, Apr 2002. Page(s): $136-146$.

[9] P.G. Datskos, N.V. Lavrik, S. Rajic. Performance of uncooled microcantilever thermal detectors, Rev. Sci. Instrum., Vol. 75, No. 4, April 2004.

[10] V.A. Fedirko, E.A. Fetisov, V.A. Bespalov. Termomechanical nanomemmrane IR-imaging sensors. Prikladnaya Fizika (Russian "Applied Physics"), Vol. 2010 / 1, p. 66-72, ISSN 19996-0948, 2010.

[11] Edwyn D. Smith. Correlated double sampling CCD video preprocessor-amplifier US patent US 4287441A, September 1,1981

[12] OPTO-SEMICONDUCTOR HANDBOOK, Chapter 04, Image Sensors, Online available from http://www.hamamatsu.com/resources/pdf/ssd/e04_handboo $\mathrm{k}$ image_sensors.pdf

[13] Visible Laser Diodes, Toshiba Product Gide, Online available from

http://photonicsguy.ca/_media/projects/405nmlaser/bce0026 _catalog.pdf

[14] Michael Bass. Handbook of Optics: Volume II - Design, Fabrication, and Testing; Sources and Detectors; Radiometry and Photometry, Third Edition. Chapter 22 - Visible Array Detectors, by T. J Tredwell. McGraw-Hill Professional, 2010.

[15] Back-thinned type CCD area image sensor, Hamamatsu, Online available from http://www.hamamatsu.com/us/en/product/category/3100/40 05/4206/4123/S9038-1002S/index.html

[16] Man-Fang Huang et al, Laser Diode for DVD Pick-up Head, Proceedings of the SPIE, Vol. 3419, pp.110-115, (1998).

[17] DVD-ROM, Combination red laser diode. Laser Diodes, Online available from www.datasheetcatalog.com. 